Sportwiss 2015 - 45:55-56

DOI 10.1007/s12662-015-0365-0

Online publiziert: 14. April 2015

๑) Springer-Verlag Berlin Heidelberg 2015

Michael Krüger

Institut für Sportwissenschaft, Universität Münster, Münster, Deutschland

\title{
Ommo Grupe und seine Vision des Sports
}

tanden von Ommo Grupe umzusetzen, was sie in Tübingen gelernt hatten.

Den Begriff „Schüler“ wollte Ommo Grupe übrigens nicht benutzen. Er vertrete keine "Schule“, hat er stets versichert. Deshalb habe er auch keine „Schüler", sondern Studenten, Mitarbeiter, Assistenten und Kollegen (und viel zu wenige „-innen“, wie er vor allem später immer bedauerte) unterschiedlichen Alters, aus unterschiedlichen Fächern und Disziplinen und mit ganz unterschiedlichen Charakteren, die voneinander lernen können, wenn sie wollen, und das gemeinsame Ziel haben - so glaubte und hoffte er -, an der Vision eines „besseren Sports“ zu arbeiten. Viele von ihnen, aber längst nicht alle, kamen 1990 in dem genannten Sammelband zu Wort.

Ommo Grupe hat als erster ordentlicher Professor und Lehrstuhlinhaber für „Theorie der Leibeserziehung “ in der Bundesrepublik Deutschland die moderne Sportpädagogik und Sportwissenschaft auf der Grundlage der Philosophischen Anthropologie begründet. Er hat es über viele Jahre geschafft, die verschiedensten wissenschaftlichen Gebiete zu einer Sportwissenschaft zusammenzuführen und dieses Fach an den Universitäten und in der akademischen Kultur insgesamt hoffähig zu machen. Er versuchte, Sport und Sportwissenschaft mit anderen gesellschaftlichen und kulturellen Bereichen und Institutionen zu vernetzen, mit Kirchen und Gewerkschaften, Bildungseinrichtungen, politischen Parteien und Jugendorganisationen. Theorie und Praxis des Sports gehörten für ihn zusammen. Er war zeit seines Lebens ein begeisterter Sportler und spielte bis zum Schluss mit seinen Tübinger Freunden und Kollegen regelmäßig Tennis. Eine besondere Leistung besteht darin, dass er sein pädagogisches Modell des Sports und der
Sportwissenschaft international verbreitete. Am eindrucksvollsten geschah dies anlässlich des Olympischen Kongresses 1972 in München. 1998 verlieh ihm das Internationale Olympische Komitee seinen Ethikpreis. Ethik und Moral im Sport standen im Mittelpunkt seines Denkens und Handelns. Das gemeinsam mit dem Theologen Dietmar Mieth in Zusammenarbeit mit den beiden großen christlichen Kirchen in Deutschland herausgegebene Lexikon der Ethik im Sport (Schorndorf 1997) ist Ausdruck dieses Bemühens um einen fairen und humanen Sport.

Was ist aus dieser Vision geworden? Wer Grupe in den letzten Jahren erlebt und begleitet hat, auch seinen letzten öffentlichen Auftritt anlässlich der Verleihung des Wissenschaftspreises des DOSB 2011 in Tübingen, wo er vom damaligen DOSB- und heutigen IOC-Präsidenten Bach besonders geehrt wurde, konnte sehen, dass er selbst an der Nachhaltigkeit seines Wirkens für den Sport zweifelte. $\mathrm{Zu}$ Grupes großem Bedauern hatte der DOSB die Carl-Diem-Plakette des DSB abgeschafft und zum Wissenschaftspreis des DOSB umbenannt. Für Grupe bedeutete das mehr als nur eine Namensänderung. Er wertete dies als eine Abkehr von den Traditionen des Sports in Westdeutschland nach 1945, die auch er selbst mit gestaltet hatte.

$\mathrm{Zu}$ Ommo Grupes Ernüchterung haben viele Entwicklungen in Sport und Sportwissenschaft beigetragen. An dieser Stelle können nicht alle genannt werden. Seine größte Enttäuschung war die Einsicht, dass Doping den Sport insgesamt und schon seit Langem weitaus stärker heimgesucht hat, als er sich das selbst vorstellen konnte oder wollte. Der Prozess der allmählichen, aber unaufhaltsamen Zerstörung seiner Vision durch Doping begann bereits in den 1970er Jahren, und Sportwissenschaften. Dort versuchten ehemalige Doktoranden und Habili- 
nach den Olympischen Spielen von Montreal. Damals glaubte er noch, dem moralischen Verfall des Sports entgegenwirken zu können. Die Grundsatzerklärung für den Spitzensport von 1977 war Ausdruck dieser Hoffnung. Mit dem Tod Birgit Dressels 1987, dann den Enthüllungen des DDR-Dopings, der Dopingaffäre Baumann direkt vor der Haustür in Tübingen und schließlich den Dopingskandalen in und um Freiburg wurde dieser Glaube nachhaltig erschüttert. Die in Teilen exzessive Kommerzialisierung und Professionalisierung des Sports beschleunigte seiner Auffassung nach die moralische Krise des Leistungs- und Spitzensports. Der Sport verlor seine Sinnmitte. Er entfernte sich von seinem ethisch-moralischen Kern.

Ein anderer, aber damit zusammenhängender Punkt hat mit der Entwicklung der Sportwissenschaft zu tun. Sie sollte und musste seiner Auffassung nach „kritisch“ sein, dem realen Sport auf die Finger sehen, ihm nahestehen und doch auf Distanz zu ihm gehen, auf jeden Fall mehr sein als affirmative, technologische Forschung für den Spitzensport. Den Bedeutungsverlust der Sportpädagogik, wie er sie verstand, nahm er mit wachsender Resignation zur Kenntnis. Zu einer kritischen und geisteswissenschaftlich fundierten Sportpädagogik und Sportwissenschaft gehörte seiner Auffassung nach ein differenziertes Wissen um die Geschichte der Leibeserziehung und des Sports. Ohne systematische sporthistorische Forschung gibt es das nicht. Sie ist jedoch in der deutschen Sportwissenschaft nach der deutschen Wiedervereinigung Schritt für Schritt marginalisiert worden. Von außen wird das Fach inzwischen nicht mehr als geisteswissenschaftliche Disziplin wahrgenommen, sondern als „anwendungsorientierte Wissenschaft" bzw. technisches Fach, wie der Historiker Wolfram Pyta schon vor Jahren in einem Beitrag für die Zeitschrift Geschichte in Wissenschaft und Unterricht (GWU 61, 2010, H $7 / 8$, S. 388) bemerkte.

Selbst wenn sich seine Vision eines besseren Sports und einer geisteswissenschaftlich orientierten Pädagogik des Sports nicht erfüllt hat, wird Ommo Grupe durch sein Leben und Wirken für die
Menschen im Sport als großes Vorbild in Erinnerung bleiben.

\section{Korrespondenzadresse}

\section{Prof. Dr. M. Krüger}

Institut für Sportwissenschaft

Universität Münster

Horstmarer Landweg 62b

48149 Münster

michael.krueger@uni-muenster.de 